Review began 02/07/2022 Review ended 02/15/2022 Published 02/18/2022

(c) Copyright 2022

Azam et al. This is an open access article distributed under the terms of the Creative Commons Attribution License CC-BY 4.0. which permits unrestricted use, distribution, and reproduction in any medium, provided the original author and source are credited.

\section{Rheumatoid Arthritis and Associated Lung Diseases: A Comprehensive Review}

Ahmad T. Azam ${ }^{1}$, Oladipo Odeyinka ${ }^{2}$, Rasha Alhashimi ${ }^{3}$, Sankeerth Thoota ${ }^{4}$, Tejaswini Ashok ${ }^{5}$, Vishnu Palyam ${ }^{6}$, Ibrahim Sange ${ }^{7}$

1. Internal Medicine, Allama Iqbal Medical College, Lahore, PAK 2. Internal Medicine, University of Ibadan College of Medicine, Ibadan, NGA 3. Internal Medicine, University of Baghdad College of Medicine, Baghdad, IRQ 4. Internal Medicine, Meenakshi Medical College Hospital and Research Institute, Kancheepuram, IND 5. Internal Medicine, Jagadguru Sri Shivarathreeshwara (JSS) Medical College, Mysore, IND 6. Internal Medicine, Jagadguru Jayadeva Murugarajendra (JJM) Medical College, Davanagere, IND 7. Research, Karamshi Jethabhai (KJ) Somaiya Medical College, Hospital and Research Center, Mumbai, IND

Corresponding author: Ahmad T. Azam, ahmadtalhaazam99@gmail.com

\begin{abstract}
Rheumatoid arthritis (RA) is a prevalent autoimmune disorder affecting $0.5-1 \%$ of the population in North America and Europe. Pulmonary manifestations in rheumatoid arthritis patients result in significant morbidity and mortality. Management of these pulmonary manifestations in RA patients causes various challenges for the physicians. This review article has discussed the current state of knowledge of these pulmonary manifestations, including interstitial lung diseases, airway-related diseases, pulmonary vasculature, and pleural involvement in RA patients. This review article has also explored various pharmacological options, including steroids, disease-modifying antirheumatic drugs (DMARDs), immunosuppressive drugs, and biologic agents. Non-pharmacological options include conservative treatment, supplemental oxygen, pulmonary rehabilitation, smoking cessation, and lung transplantation.
\end{abstract}

Categories: Internal Medicine, Pulmonology, Rheumatology

Keywords: rheumatoid arthritis, rheumatoid disorder, interstitial lung disease, complication of rheumatoid arthritis, progressive interstitial lung disease

\section{Introduction And Background}

Rheumatoid arthritis (RA) is one of the most prevalent chronic inflammatory diseases characterized by progressive autoimmune and inflammatory damage to cartilage, bones and synovial lining of the joints eventually resulting in the severe disability of the patient [1,2]. The prevalence of RA in North America and Europe has been reported to be around $0.5-1 \%$ [1]. However, the prevalence and incidence of RA have been a recent topic of debate. Several studies reported that the incidence of rheumatoid arthritis decreased during the latter half of the 20th century. However, Myasoedova et al. reported that the incidence of RA has been on the rise again since 1995 [1,3]. According to a recent observational study by Hunter et al., the prevalence of RA has increased from 2004 to 2014 [4]. The female sex is predominantly more affected than their male counterpart [1]. Other major risk factors include old age, smoking, family history of the disease, and obesity [5]. RA can typically affect any age group but is more common among people aged 30-50 [5].

Pathophysiology of RA involves infiltration of T-lymphocytes, B-lymphocytes and monocytes in the synovial membrane of different joints of the body [6]. Pro-inflammatory cytokines including interleukin-1 (IL-1), tumor necrosis factor (TNF), and interleukin-6 (IL-6) released by macrophages mediate the damage to the surrounding bone and cartilage [7]. Activation of endothelial cells and proliferation of fibroblast-like cells in the synovial membrane result in neovascularization and pannus formation, respectively [8]. The etiology of rheumatoid arthritis is multifactorial [9]. Genetic susceptibility plays a major role and increases the risk of developing RA threefold to ninefold. HLA-DRB1 (Human Leukocyte Antigen DR Beta 1) region is strongly associated with anti-cyclic citrullinated peptide antibodies (ACPA) positive RA [10]. Several triggers, including smoking, infections and dust particles, have been studied for their role in causing RA [9]. However, smoking has been the most consistent risk factor associated with RA [11]. RA classically presents as a symmetrical polyarthritis primarily affecting small joints of hands and feet. The patients of RA mostly present with the complaint of pain and swelling in multiple joints of the body that is accompanied by morning stiffness that stays for more than 30 minutes but gets better with activity [12]. When left untreated, RA can cause joint deformities and atlantoaxial subluxation, which can eventually lead to severe disability of the patient [13]. Extra-articular manifestations (EAM) are common in the later stages of the disease and involve rheumatoid nodules near bony prominences, fever, weight loss, and myalgias [12]. Pulmonary manifestations include pleural effusions, interstitial lung diseases, and pleuritis [14]. Cardiovascular manifestations include pericarditis, myocarditis, coronary vasculitis, and enhanced risk of congestive heart failure [15]. Neurological manifestations include median nerve compression, mononeuritis multiplex, and entrapment neuropathy secondary to synovitis [16]. Other EAM includes osteoporosis, Felty's syndrome, glomerulonephritis, and scleritis [17]. Early diagnosis and treatment of RA have shown promising clinical outcomes for the patients, including a higher possibility of drug-free remission and decreased damage to the joints [18]. The diagnosis of RA is primarily clinical as there are no set diagnostic criteria established yet. 


\section{Cureus}

However, 2010 ACR/EULAR (American College of Rheumatology/ European League Against Rheumatism) Classification criteria for RA can help the physicians establish the diagnosis [19]. X-ray, ultrasound, and magnetic resonance imaging (MRI) can assess the severity of damage to the joints [20]. Serologic testing for autoantibodies, including ACPA and rheumatoid factor (RF), can be helpful in the diagnosis but up to $30 \%$ of RA are negative for these autoantibodies [12]. Treatment should be initiated as early as possible after a diagnosis of RA has been made. The primary treatment goals involve decreasing pain and swelling in the joints, reducing radiological disease severity, improving the patient's quality of life, and controlling extraarticular manifestation of rheumatoid arthritis [21]. Disease-modifying antirheumatic drugs (DMARDs) are the most common treatment options for RA patients. Monotherapy with methotrexate and corticosteroids is recommended as the first line of treatment for RA patients [21]. Combination therapy with two or more DMARDs can be used in case of failure of monotherapy. Biologic DMARDs, including anti-TNF agents (adalimumab, golimumab) can be added to therapy if disease remission is not achieved with synthetic DMARDs. Nonsteroidal anti-inflammatory drugs (NSAIDs) and corticosteroids help decrease inflammation [21]. Pulmonary manifestations are one of the most challenging complications faced during the management of RA patients. RA-associated interstitial lung diseases (RA-ILD), pleural effusion, pleuritis, bronchiectasis, pulmonary vascular diseases, and drug-associated lung complications are major pulmonary manifestations caused by RA [22]. It is important to probe into the challenges faced by physicians and patients due to high morbidity and mortality due to pulmonary complications in RA. This review article aims to review the spectrum of pulmonary manifestations in RA, discuss the latest pharmacological and non-pharmacological options for managing RA-associated lung diseases and discuss the challenges physicians face in managing this morbidity.

\section{Review}

Rheumatoid arthritis is associated with a wide range of pulmonary manifestations. Some of the major RAassociated lung diseases are discussed below:

\section{RA-associated interstitial lung diseases}

Interstitial lung disease (ILD) is one of the most prevalent and well-studied pulmonary manifestations of rheumatoid arthritis [23]. Most commonly diagnosed RA-ILDs include usual interstitial pneumonia (UIP) and non-specific interstitial pneumonia (NSIP) [24]. However, diffuse alveolar damage, organizing pneumonia $(\mathrm{OP})$, acute interstitial pneumonia, and obliterative bronchiolitis have also been reported in RA patients.

The pathogenesis of RA-ILD is still not well understood; however, genetics and environmental factors play a significant role in the manifestation of ILD in RA patients [14]. Smoking, advanced age, male sex, high-titer anti-cyclic citrullinated peptide antibodies, high-titer rheumatoid factor, and genetics are major risk factors of RA-ILDs (Figure 1). 


\section{Cureus}

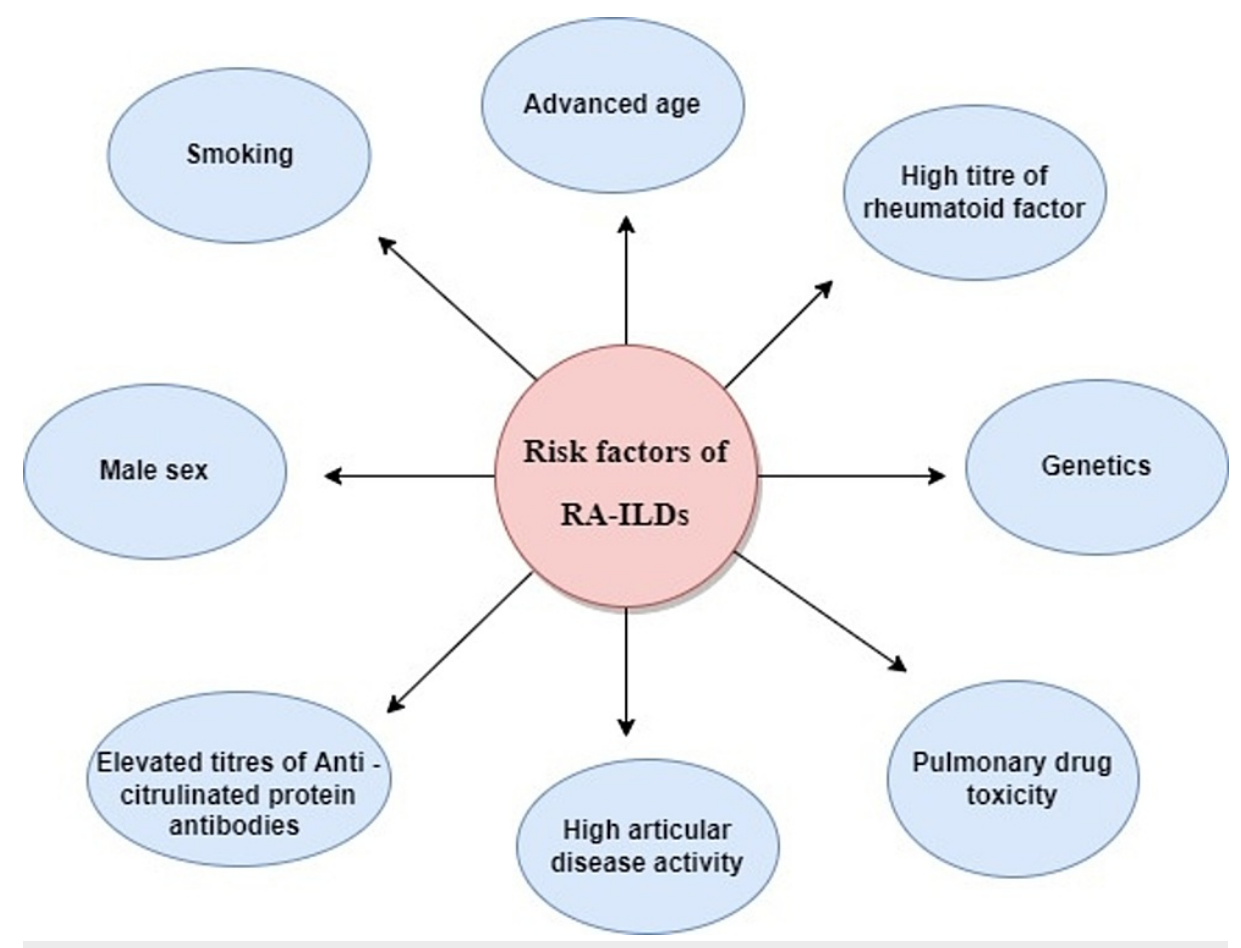

FIGURE 1: Risk factors of RA-ILD

RA - Rheumatoid Arthritis, ILD- Interstitial Lung Diseases

HLA-DRB1, HLA-DR4, and HLA-B40 are some of the significant HLA variants that play their role in the manifestation of ILDs in RA patients [25]. Smoking has been found to play an important role in triggering the immune reaction to citrulline-modified proteins by interacting with HLA-DR shared epitope (SE) genes [26]. A population-based case-control study was conducted in Sweden in 2006 that included 1544 participants (913 cases and 631 controls). It was found in this study that the previous history of smoking and the presence of two copies of HLA-DR SE genes enhanced the risk for rheumatoid arthritis by 21-fold as compared to non-smokers with no SE genes [26]. Several epidemiological studies have also reported an undeniable association between smoking and RA-ILDs [27]. Lung parenchymal and airway damage due to environmental exposure results in enhanced citrullination of proteins [28]. This initiates an inflammatory process and activation of cytokines, chemokines and growth factors, such as tumor necrosis factor (TNF), interleukins (IL), and vascular endothelial growth factor (VEGF) in a genetically predisposed individual [29]. Proliferation and differentiation of fibroblasts cause matrix metalloproteinases (MMP) hyperactivity and enhanced deposition of extracellular matrix (ECM); this results in the development of ILD and pulmonary fibrosis [27]. However, Zhang et al. recently reported that Interleukin-17 (IL-17) can have a major role in the pathogenesis of RA-ILD as lung biopsies of RA-ILD patients had demonstrated higher IL-17 expression in areas of fibrosis [30].

Clinical features of RA-ILDs include cough of insidious onset, external dyspnea, fatigue, and generalized weakness. Symptoms generally progress slowly, but the rate of progression varies from one patient to another [27]. It also varies among different histological subtypes [24]. A study was conducted by Kim et al. at the University of California, San Francisco and the Mayo Clinic, United States of America (USA) between 2001 and 2008. The study included a sample population of 82 patients with RA-ILD, which was followed for a median duration of 3.9 yrs (range 0.3-7.5 yrs). RA-UIP cohort had a median survival time of 3.2 years which was worse than the non-UIP cohort, which had a median survival time of 6.6 years. It concluded that UIP has a faster rate of progression and worse survival rate than other histological subtypes of ILD in RA patients [31].

Imaging plays a critical role in the diagnosis of RA-ILDs. Chest radiograph and high-resolution computed tomography (HRCT) are the initial investigations to identify the distribution and patterns of interstitial parenchymal abnormalities. HRCT has significantly higher diagnostic sensitivity as compared to chest radiographs. A study was conducted by Dawson et al. in a sample population of 150 patients with RA; HRCT evidence of ILD was observed in 28 patients (19\%). On the other hand, findings consistent with ILD were observed in chest radiographs in four patients $(<3 \%)$. This concluded that chest radiograph is not independently predictive investigation in patients with high suspicion of RA-ILD and abnormal chest radiographic findings warrant the need for HRCT to rule out RA-ILD in such patients [32]. 
The most common radiological feature in the UIP is subpleural and basilar predominant reticular abnormalities [33]. Honeycombing and traction bronchiectasis are also present, but ground-glass opacification is usually absent [33]. On the other hand, NSIP is characterized by ground-glass opacification with minimal honeycombing or architectural distortion [34]. Pulmonary function tests (PFTs) can be helpful diagnostic modality especially in the absence of symptoms. In patients with RA-ILD, PFTs may show a pattern of restrictive lung disease with a reduced diffusing capacity of the lung for carbon monoxide (DLCO) [35]. Bronchoalveolar lavage (BAL) in patients with RA-ILD yields non-specific results. Lymphocytosis is the most common finding in most patterns except in UIP. Neutrophilia is more typical in patients with UIP patterns [36]. Bronchoalveolar lavage and surgical lung biopsy can be helpful diagnostic modalities if the diagnosis is uncertain or findings on HRCT are atypical [27].

Early detection of RA-ILD results in a significant decline in disease progression [37]. A study was conducted by Gochuico et al. involving a sample population of 64 adults with RA and 10 adults with rheumatoid arthritis-related pulmonary fibrosis (RAPF). Twenty-one of 64 (33\%) RA patients without pulmonary symptoms had preclinical ILD detectable by HRCT. Follow-up of this sample population mean follow-up time period being 2.5 years, reported that radiological disease detectable on HRCT progressed in 12 of 21 subjects (57\%). This study concluded that asymptomatic pulmonary manifestations, including ILDs that are only detectable by HRCT, can be progressive in patients with RA [37].

Given the significant prevalence of pulmonary manifestations in RA, physicians should have a low threshold to proceed with a detailed evaluation of pulmonary symptoms in RA patients [38]. The prognosis varies with different histological subtypes, and studies have demonstrated that the UIP subtype exhibits the worst prognosis of all the RA-ILDs [39]. A study was conducted by Olson et al. to ascertain mortality rates from RAILDs in the United States from 1988 through 2004, demonstrating that overall mortality from RA is declining; however, mortality from RA-ILD is on the rise, especially in older age groups [40]. In another cohort study by Bongartz et al., which included a sample population of 582 patients with RA and 603 control subjects without RA, it was indicated that there was a $13 \%$ increased risk of mortality due to ILD in RA patients as compared to the general population. This study also observed that the risk of mortality for RA patients with ILD was threefold higher than in RA patients without ILD [41].

\section{RA-related airway diseases}

Most commonly seen airway-related manifestations in RA patients include bronchiectasis, bronchiolitis, and cricoarytenoid arthritis [42]. It has been observed that 39 to $60 \%$ of RA patients develop airway-related morbidities in their lifetime [43]. HRCT and PFTs can be very helpful in diagnosing large airway involvement, although HRCT has a tremendous diagnostic sensitivity for small airway involvement in RA patients [42].

Large airway abnormalities include bronchiectasis and cricoarytenoid arthritis. Bronchiectasis has been reported in around 30 percent of RA patients [27]. It typically presents with insidious cough and productive sputum [44]. Various theories have been proposed regarding the pathogenesis of bronchiectasis in RA patients. Chronic suppurative infections and the use of DMARDs in the treatment of RA are the leading causes of bronchiectasis in RA patients. A longitudinal cohort study was conducted by Geri et al. between 2000 and 2009 that included 47 RA patients with bronchiectasis. After a mean follow-up per patient of $4.3 \pm$ 3.1 years, it was observed that the use of biological agents in the treatment of RA and sputum colonization by bacterial organisms are independent risk factors for lower respiratory tract infection [45]. Cricoarytenoid joint arthritis presents with hoarseness, throat pain, dyspnea, and stridor due to midline adduction of the vocal cords. It primarily occurs due to synovial thickening of the cricoarytenoid joint, resulting in continuous cartilage erosion. HRCT of the neck can diagnose the condition before the appearance of clinical symptoms. Complications include acute stridor or obstructive respiratory failure [46].

Small airway abnormalities due to RA include follicular and constrictive bronchiolitis [22]. Follicular bronchiolitis may present with exertional dyspnea, nonproductive cough, and wheezing. HRCT is the mainstay of diagnosis and can demonstrate findings before clinical symptoms. Follicular bronchiolitis is characterized by hyperplasia of bronchial-associated lymphoid tissue. Presence of centrilobular peribronchial nodules less than 3mm in size is detectable on HRCT [47]. Unlike other rheumatoid pulmonary manifestations, obliterative bronchiolitis usually has a more severe and acute clinical presentation. It may present with cough, bronchorrhea, and rapidly progressive dyspnea without systemic symptoms. Constrictive bronchiolitis is characterized by damaged airway epithelium resulting in airflow obstruction. HRCT findings include mosaic attenuation, bronchial wall thickening, and centrilobular nodules [48].

\section{RA-associated pleural diseases}

Pleuritis and pleural effusion are the most prevalent pleural manifestations observed in RA patients. The pleural disease in RA patients can occur before or alongside the first clinical signs of joint involvement. Pleural disease is primarily silent in RA patients with symptomatic disease present in only $3-5 \%$ of the patients [49]. Large pleural effusions can present with cough, dyspnea, chest pain, and fever [50]. 
produced by plural mononuclear cells which contribute to the formation of immune complexes [51]. These immune complexes damage the capillary endothelium and increase the capillary permeability of pleural space. Other proposed mechanisms include high protein content in rheumatoid pleural effusion and blockage of lymphatic drainage in inflamed parietal pleura contributes towards the formation of rheumatoid pleural effusion [51].

Ultrasound-guided thoracocentesis is usually considered first-line investigation in patients with high suspicion of the disease. Pleural fluid in RA usually has low $\mathrm{pH}(<7.3)$ and glucose levels $(<50 \mathrm{mg} / \mathrm{dL})[51]$. Rheumatoid factor is usually a characteristic finding in the pleural fluid analysis of RA patients but its absence doesn't rule out the possibility of the disease. Cytological analysis of pleural effusion reveals multinucleated giant cells, macrophage predominance, and granulomatous debris. RA pleural effusions contain a high titer of RF, raised adenosine deaminase levels, decreased Complement total (CH50) levels [50]. Medical thoracoscopy and pleural biopsy are required if the diagnosis is still unclear on thoracocentesis. It has been reported that severe or untreated pleural effusion can eventually result in fibrothorax and lung restriction [49]. The risk of empyema in RA patients is still not well studied; however, it has been hypothesized that patients treated with immunosuppressive therapy had a higher risk of developing empyema [52]. However, clinical trials with a large sample population are not available to support this hypothesis.

\section{Other RA-associated pulmonary manifestations}

Other uncommon RA-associated pulmonary manifestations include rheumatoid pulmonary nodules, rheumatoid pulmonary vasculitis, and drug-induced pulmonary complications [53]. Rheumatoid pulmonary nodules can occur in RA patients when exposed to inorganic dust, including asbestos, silica, and coal. A prospective study by Zrour et al. with a sample population of 75 RA patients undergoing HRCT reported rheumatoid pulmonary nodules in $4 \%$ of patients [54]. The disease was clinically silent in $4 \%$ of these cases. HRCT is the modality of choice to detect rheumatoid nodules as they can be missed in the regular chest radiographs [54]. There is no recent data on the incidence of this manifestation in RA patients [54]. Rheumatoid pulmonary vasculitis can most commonly manifest as pulmonary angiitis in 1\% of RA patients [55]. It presents along with cutaneous vasculitis and vasculitis neuropathy [55]. Drug-induced pulmonary complications of different drugs used by RA patients are discussed in the management section below.

\section{Updated management guidelines}

Early treatment should be started in patients showing clinical or radiologic manifestations of the disease. The disease severity and rate of progression of the disease in RA-ILD patients can be used to identify the need to start or augment the therapy to manage pulmonary manifestations of RA [27]. Treatment includes supportive measures and the use of anti-inflammatory agents to reduce the inflammation involved in the pathogenesis of the disease.

\section{Conservative Management}

Conservative management of RA-ILD patients is usually considered in the case of mild disease or patients with multiple comorbidities and advanced age due to contraindication of pharmacological management [27]. Due to the well-documented role of smoking in the pathogenesis of RA-ILD, it is important to provide smoking cessation counseling to the patients and educate them regarding its implications in the worsening of the disease [27]. Supplemental oxygen has also proven to be an important part of a palliative protocol for patients with severe disease [56]. Several studies have shown that supplemental oxygen improves endurance time, walking distance, and dyspnea in ILD patients [56]. However, there are no clinical trials available with a sufficient patient population to support this evidence. Pulmonary rehabilitation has shown benefits in improving pulmonary symptoms in patients with idiopathic pulmonary fibrosis (IPF), so it can be theorized that it will help RA-ILD patients as well. However, functional limitations of RA patients restrict their ability to perform exercises involved in this intervention [57]. This warrants the need for tailored pulmonary rehabilitation protocol for RA-ILD patients. Psychosocial support, Pneumococcal, and Influenza vaccination are other conservative interventions recommended for RA-ILD patients [58].

Pharmacological Management

Several treatment options are recommended based on updated clinical studies. Below, we discuss the rationale behind each recommendation for pharmacological management of RA-ILD patients.

Immunosuppressive Drugs

Glucocorticoids are generally considered the first line of management for patients with RA-ILDs. It is recommended to start with oral Prednisone at a daily dose of $0.5 \mathrm{mg} / \mathrm{kg}$ with subsequent tapering based on the clinical response of the patient [59]. In a retrospective study by Song et al. on 84 patients with RA-related UIP, $50 \%$ of the patients showed improvement in their clinical symptoms when treated with glucocorticoids alone or in combination with DMARDs [60]. 


\section{Cureus}

It is suggested that the survival rate of patients with ILD increases with the use of another immunosuppressive drug, Azathioprine. A prospective, double-blind, randomized, placebo-controlled study was done by Raghu et al. involving 27 newly diagnosed patients with IPF. Fourteen patients were treated with Azathioprine and Prednisone and 13 with Prednisone alone. The study reported an improved survival rate in a cohort treated with a combined regimen of steroids and Azathioprine [61]. However, a recent trial, "Prednisone, Azathioprine, N-acetylcysteine: A Study That Evaluates Response in Idiopathic Pulmonary Fibrosis (PANTHER-IPF study)" reported that mortality is increased in the patients treated with Azathioprine and Prednisone. This study had to be discontinued prematurely due to the high mortality rate in patients treated with Azathioprine and Prednisone [62].

Mycophenolate mofetil (MMF) is another potentially helpful immunosuppressive drug with encouraging results in several retrospective studies. MMF inhibits inosine monophosphate dehydrogenase (IMPDH), eventually hindering de novo purine biosynthesis. It diminishes $\mathrm{B}$ and $\mathrm{T}$ lymphocyte proliferation, reducing inflammation [63]. In a study of 125 patients with connective tissue disease-related ILD treated with MMF by Fischer et al. in 2013, an analysis of 18 patients with RA-ILD showed that patients treated with MMF for a median of 897 days had improved forced vital capacity (FVC). Less than $10 \%$ of the patients in the study discontinued the treatment due to adverse effects of the drug [64]. In a meta-analysis by Tzouvelekis et al. with a sample population of 69 patients with systemic sclerosis-associated interstitial lung disease, it was reported that the use of MMF has improved disease stabilization and prevented forced vital capacity (FVC) decline [65].

DMARDs

A significant hurdle in the treatment of RA-ILD is the fact that many potential pharmacological options like DMARDs and biologic agents have proven record to cause pulmonary toxicity. Methotrexate (MTX) and antiTNF agents should be used with caution in patients with RA-ILD [66]. Non-biologic DMARDs, including methotrexate and Leflunomide (LEF), have well-demonstrated ILD promoting effects, due to which they are not considered a primary therapeutic option in RA-ILD patients. A meta-analysis by Conway et al. encompassing 22 studies with 8,584 participants reported that MTX is associated with an increased risk of pneumonitis and other respiratory infections [66]. It is recommended to get pulmonary functions tests done before initiating MTX as most patients develop pulmonary complications within the first months of initiation of treatment [67].

\section{Biologics}

Anti-TNF agents have both antifibrotic and profibrotic properties that make their use controversial in the treatment of RA-ILDs [68]. Use of adalimumab, golimumab, infliximab, and etanercept has been linked with new-onset or exacerbation of ILDs [69-72]. In one study by Perez-Alvarez et al., 122 reported cases of newonset or worsened ILD in the setting of anti-TNF use were evaluated. A total of 108 cases used in the study were of RA patients. 97\% of cases of ILD reported in the study were linked with the use of anti-TNF agents including etanercept and infliximab [73]. In contrast, a prospective observational study by Dixon et al. involving a sample population of 367 patients with RA-ILD demonstrated that the mortality rate was not increased after treatment with anti-TNF agents compared with traditional immunomodulatory drugs (Table 1) [74]. Several cases have been reported in which stabilization of pulmonary function was seen in patients using anti-TNF agents [75]. However, the evidence available regarding the efficacy of anti-TNF agents in RAILD patients is insufficient. 


\section{Cureus}

\begin{tabular}{|c|c|c|c|c|}
\hline References & Design & $\begin{array}{l}\text { Number of } \\
\text { participants }\end{array}$ & Methods & Conclusion \\
\hline $\begin{array}{l}\text { Klareskog } \\
\text { et al. (2006) } \\
\text { [26] }\end{array}$ & $\begin{array}{l}\text { Population- } \\
\text { based case- } \\
\text { control study }\end{array}$ & $\begin{array}{l}1544(913 \\
\text { cases }+631 \\
\text { controls })\end{array}$ & $\begin{array}{l}\text { Cases were individuals aged } 18-70 \text { years with } \\
\text { newly diagnosed RA. There was a random } \\
\text { selection of controls from the Swedish National } \\
\text { Population Registry. }\end{array}$ & $\begin{array}{l}\text { Previous history of smoking and the } \\
\text { presence of two copies of HLA-DR SE } \\
\text { genes enhanced the risk for rheumatoid } \\
\text { arthritis by } 21 \text {-fold. }\end{array}$ \\
\hline $\begin{array}{l}\text { Kim et al. } \\
\text { (2010) [31] }\end{array}$ & Cohort study & 82 & $\begin{array}{l}82 \text { patients with RA-ILD (identified } \\
\text { retrospectively) participated in the study } \\
\text { between } 2001 \text { and } 2008 .\end{array}$ & $\begin{array}{l}\text { UIP has a faster rate of progression than } \\
\text { other histological subtypes of ILD in RA } \\
\text { patients. }\end{array}$ \\
\hline $\begin{array}{l}\text { Dawson et } \\
\text { al. (2001) } \\
\text { [32] }\end{array}$ & Cohort study & 150 & 150 patients with RA & $\begin{array}{l}19 \% \text { of patients but evidence of ILD on } \\
\text { chest radiograph was seen in } 3 \% \text {. }\end{array}$ \\
\hline $\begin{array}{l}\text { Gochuico et } \\
\text { al. (2008) } \\
\text { [37] }\end{array}$ & Cohort study & 74 & $\begin{array}{l}64 \text { adults with RA and } 10 \text { adults with rheumatoid } \\
\text { arthritis-related pulmonary fibrosis. }\end{array}$ & $\begin{array}{l}33 \% \text { of RA patients without pulmonary } \\
\text { symptoms had preclinical ILD detectable } \\
\text { by HRCT. }\end{array}$ \\
\hline $\begin{array}{l}\text { Bongartz et } \\
\text { al. }(2010) \\
\text { [41] }\end{array}$ & Cohort study & $\begin{array}{l}1185 \text { ( } 582 \\
\text { cases and } \\
603 \text { control) }\end{array}$ & $\begin{array}{l}582 \text { RA patients } 603 \text { control subjects were } \\
\text { followed for a mean of } 16.4 \text { and } 19.3 \text { years, } \\
\text { respectively. }\end{array}$ & $\begin{array}{l}\text { Risk of mortality for RA patients with ILD } \\
\text { was threefold higher than in RA patients } \\
\text { without ILD. }\end{array}$ \\
\hline $\begin{array}{l}\text { Song et al. } \\
\text { (2103) [60] }\end{array}$ & $\begin{array}{l}\text { Retrospective } \\
\text { study }\end{array}$ & 84 & 84 patients with RA-UIP & $\begin{array}{l}50 \% \text { of the patients showed improvement } \\
\text { in their clinical symptoms when treated } \\
\text { with glucocorticoid alone or in combination } \\
\text { with DMARDs. }\end{array}$ \\
\hline $\begin{array}{l}\text { Dixon et al. } \\
\text { (2010) [74] }\end{array}$ & $\begin{array}{l}\text { Prospective } \\
\text { observational } \\
\text { study }\end{array}$ & 367 & 367 patients with RA-ILD & $\begin{array}{l}\text { Mortality was not increased after treatment } \\
\text { with anti-TNF agents compared with } \\
\text { traditional DMARDs. }\end{array}$ \\
\hline
\end{tabular}

\section{TABLE 1: Summary of studies demonstrating pathophysiology and management of rheumatoid arthritis-associated interstitial lung diseases}

RA - Rheumatoid Arthritis, DMARDS - Disease-Modifying Antirheumatic Drugs, ILD - Interstitial Lung Diseases, UIP - Usual Interstitial Pneumonia, TNF Tumor Necrosis Factor, HRCT - High-Resolution Computed Tomography, HLA - Human Leukocyte Antigen, SE - Shared Epitope

Rituximab is a monoclonal antibody against CD20 B-cell marker and is currently being studied as a potential treatment for RA-ILD patients. Several studies have hypothesized that there can be a role of B-cells in the pathogenesis of RA-ILD. Atkins et al. reported significant follicular B cell hyperplasia in samples of open lung biopsies of RA-ILD patients [76]. Small observational studies and case reports have shown promising results regarding the efficacy of rituximab, but they are still inconclusive. Becerra et al. did a cohort study on 264 RA patients being treated with rituximab; 38 of them had lung involvement before initiation of treatment. No evidence of progression of lung symptoms was found in RA-ILD patients after a median follow-up period of 2.5 years, but no improvement was also seen in their pulmonary symptoms [77]. Clinical trials with a larger cohort of patients are required to delineate the safety and efficacy of rituximab in this subset of the population.

\section{Surgical intervention}

Lung Transplant

A lung transplant can be considered for young patients with advanced refractory disease. However, it is contraindicated in patients with advanced age, multiple comorbidities, immobility, and other extra-articular manifestations. A systematic review by Richardson and Singer reported a 5 -year survival rate from $46 \%$ to $76 \%$ in systemic sclerosis ILD patients who received a lung transplant [78].

\section{Future implications}

New treatment options are being studied, including pirfenidone, an antifibrotic agent. A study by Wu et al. has demonstrated antifibrotic effects of pirfenidone in RA-ILD lung specimens. A transition of fibroblast to myofibroblast was inhibited by down-regulation of Smad3-ATF3 signaling [79]. Genome-wide association studies are being conducted on RA-ILD patients, which will provide a deeper insight into the pathogenesis of the disease [58]. Researchers are trying to find accurate and clinically practical biomarkers which can enable 
clinicians to make an early diagnosis of the disease, eventually resulting in early initiation of treatment [80].

\section{Limitations}

Our literature review does not provide a standard treatment algorithm that can be followed for RA-ILD patients due to the lack of randomized controlled trials (RCTs) in this subset of the population. Treatment protocols need to be individualized for each patient. This study does not address the growing importance of unique biomarkers that physicians can use for an early diagnosis of RA-ILD patients.

\section{Conclusions}

This review article discussed the spectrum of pulmonary manifestations in RA patients, updated management guidelines, and challenges faced by the physicians during the management of such patients. The clinical implication of this review article is to summarize the current state of knowledge of pulmonary manifestations in RA patients which will help the physicians better understand the disease and novel treatment protocols for RA-ILD patients. We discussed the challenges faced by the clinicians while managing such patients and how they can be tackled through a tailored management approach for each patient. Major pulmonary manifestations include ILDs, airway-related diseases, and pleural diseases, including pleuritis and pleural effusion. Pathogenesis of RA-ILD has been associated with genetics and environmental exposures, including smoking. Chest radiograph, HRCT, and PFTs are considered major diagnostic modalities. Treatment is tailored for each patient depending on various factors and is begun by nonpharmacological measures such as smoking cessation, supplemental oxygen therapy, and chest physiotherapy. As per the studies reviewed above, steroids are generally the first line of treatment. Other immunosuppressive drugs and biologic agents can be added to the regimen after carefully considering the patient's previous medical history. Lastly, we recommend more future studies, primarily controlled therapeutic trials with a sufficient patient population to identify novel and safe therapeutic options in this patient population.

\section{Additional Information \\ Disclosures}

Conflicts of interest: In compliance with the ICMJE uniform disclosure form, all authors declare the following: Payment/services info: All authors have declared that no financial support was received from any organization for the submitted work. Financial relationships: All authors have declared that they have no financial relationships at present or within the previous three years with any organizations that might have an interest in the submitted work. Other relationships: All authors have declared that there are no other relationships or activities that could appear to have influenced the submitted work.

\section{References}

1. Myasoedova E, Crowson CS, Kremers HM, Therneau TM, Gabriel SE: Is the incidence of rheumatoid arthritis rising?: results from Olmsted County, Minnesota, 1955-2007. Arthritis Rheum. 2010, 62:1576-1582. 10.1002/art.27425

2. Smolen JS, Aletaha D, McInnes IB: Rheumatoid arthritis. Lancet. 2016, 388:2023-2038. 10.1016/S01406736(16)30173-8

3. Linos A, Worthington JW, O'Fallon WM, Kurland LT: The epidemiology of rheumatoid arthritis in Rochester, Minnesota: a study of incidence, prevalence, and mortality. Am J Epidemiol. 1980, 111:87-98. 10.1093/oxfordjournals.aje.a112878

4. Hunter TM, Boytsov NN, Zhang X, Schroeder K, Michaud K, Araujo AB: Prevalence of rheumatoid arthritis in the United States adult population in healthcare claims databases, 2004-2014. Rheumatol Int. 2017, 37:1551-1557. 10.1007/s00296-017-3726-1

5. Deane KD, Demoruelle MK, Kelmenson LB, Kuhn KA, Norris JM, Holers VM: Genetic and environmental risk factors for rheumatoid arthritis. Best Pract Res Clin Rheumatol. 2017, 31:3-18. 10.1016/j.berh.2017.08.003

6. Brennan FM, McInnes IB: Evidence that cytokines play a role in rheumatoid arthritis . J Clin Invest. 2008, 118:3537-3545. 10.1172/JCI36389

7. Dayer JM, Beutler B, Cerami A: Cachectin/tumor necrosis factor stimulates collagenase and prostaglandin E2 production by human synovial cells and dermal fibroblasts. J Exp Med. 1985, 162:2163-2168. 10.1084/jem.162.6.2163

8. Bartok B, Firestein GS: Fibroblast-like synoviocytes: key effector cells in rheumatoid arthritis . Immunol Rev. 2010, 233:233-255. 10.1111/j.0105-2896.2009.00859.x

9. Arleevskaya MI, Kravtsova OA, Lemerle J, Renaudineau Y, Tsibulkin AP: How rheumatoid arthritis can result from provocation of the immune system by microorganisms and viruses. Front Microbiol. 2016, 7:1296. 10.3389/fmicb.2016.01296

10. Lenz TL, Deutsch AJ, Han B, et al.: Widespread non-additive and interaction effects within HLA loci modulate the risk of autoimmune diseases. Nat Genet. 2015, 47:1085-1090. 10.1038/ng.3379

11. Bang SY, Lee KH, Cho SK, Lee HS, Lee KW, Bae SC: Smoking increases rheumatoid arthritis susceptibility in individuals carrying the HLA-DRB1 shared epitope, regardless of rheumatoid factor or anti-cyclic citrullinated peptide antibody status. Arthritis Rheum. 2010, 62:369-377. 10.1002/art.27272

12. Aletaha D, Smolen JS: Diagnosis and management of rheumatoid arthritis: a review . JAMA. 2018, 320:13601372. 10.1001/jama.2018.13103

13. Zhang T, Pope J: Cervical spine involvement in rheumatoid arthritis over time: results from a meta-analysis . 
Arthritis Res Ther. 2015, 17:148. 10.1186/s13075-015-0643-0

14. Wang D, Zhang J, Lau J, Wang S, Taneja V, Matteson EL, Vassallo R: Mechanisms of lung disease development in rheumatoid arthritis. Nat Rev Rheumatol. 2019, 15:581-596. 10.1038/s41584-019-0275-X

15. Crowson CS, Liao KP, Davis JM 3rd, et al.: Rheumatoid arthritis and cardiovascular disease . Am Heart J. 2013, 166:622-628.e1. 10.1016/j.ahj.2013.07.010

16. DeQuattro K, Imboden JB: Neurologic manifestations of rheumatoid arthritis. Rheum Dis Clin North Am. 2017, 43:561-571. 10.1016/j.rdc.2017.06.005

17. Das S, Padhan P: An overview of the extraarticular involvement in rheumatoid arthritis and its management. J Pharmacol Pharmacother. 2017, 8:81-86. 10.4103/jpp.JPP_194_16

18. Lin YJ, Anzaghe M, Schülke S: Update on the pathomechanism, diagnosis, and treatment options for rheumatoid arthritis. Cells. 2020, 9:880. 10.3390/cells9040880

19. Kay J, Upchurch KS: ACR/EULAR 2010 rheumatoid arthritis classification criteria. Rheumatology (Oxford). 2012, 51:vi5-vi9. 10.1093/rheumatology/kes279

20. D'Agostino MA, Terslev L, Wakefield R, et al.: Novel algorithms for the pragmatic use of ultrasound in the management of patients with rheumatoid arthritis: from diagnosis to remission. Ann Rheum Dis. 2016, 75:1902-1908. 10.1136/annrheumdis-2016-209646

21. Fraenkel L, Bathon JM, England BR, et al.: 2021 American College of Rheumatology guideline for the treatment of rheumatoid arthritis. Arthritis Rheumatol. 2021, 73:1108-1123. 10.1002/art.41752

22. Yunt ZX, Solomon JJ: Lung disease in rheumatoid arthritis. Rheum Dis Clin North Am. 2015, 41:225-236. 10.1016/j.rdc.2014.12.004

23. Norton S, Koduri G, Nikiphorou E, Dixey J, Williams P, Young A: A study of baseline prevalence and cumulative incidence of comorbidity and extra-articular manifestations in RA and their impact on outcome. Rheumatology (Oxford). 2013, 52:99-110. 10.1093/rheumatology/kes262

24. Nakamura Y, Suda T, Kaida Y, et al.: Rheumatoid lung disease: prognostic analysis of 54 biopsy-proven cases. Respir Med. 2012, 106:1164-1169. 10.1016/j.rmed.2012.04.004

25. McInnes IB, Schett G: The pathogenesis of rheumatoid arthritis . N Engl J Med. 2011, 365:2205-2219. 10.1056/NEJMra1004965

26. Klareskog L, Stolt P, Lundberg K, et al.: A new model for an etiology of rheumatoid arthritis: smoking may trigger HLA-DR (shared epitope)-restricted immune reactions to autoantigens modified by citrullination. Arthritis Rheum. 2006, 54:38-46. 10.1002/art.21575

27. Kadura S, Raghu G: Rheumatoid arthritis-interstitial lung disease: manifestations and current concepts in pathogenesis and management. Eur Respir Rev. 2021, 30:210011. 10.1183/16000617.0011-2021

28. Valesini G, Gerardi MC, Iannuccelli C, Pacucci VA, Pendolino M, Shoenfeld Y: Citrullination and autoimmunity. Autoimmun Rev. 2015, 14:490-497. 10.1016/j.autrev.2015.01.013

29. Rantapää-Dahlqvist S, de Jong BA, Berglin E, et al.: Antibodies against cyclic citrullinated peptide and IgA rheumatoid factor predict the development of rheumatoid arthritis. Arthritis Rheum. 2003, 48:2741-2749. 10.1002/art.11223

30. Zhang J, Wang D, Wang L, et al.: Profibrotic effect of IL-17A and elevated IL-17RA in idiopathic pulmonary fibrosis and rheumatoid arthritis-associated lung disease support a direct role for IL-17A/IL-17RA in human fibrotic interstitial lung disease. Am J Physiol Lung Cell Mol Physiol. 2019, 316:487-497. 10.1152/ajplung.00301.2018

31. Kim EJ, Elicker BM, Maldonado F, et al.: Usual interstitial pneumonia in rheumatoid arthritis-associated interstitial lung disease. Eur Respir J. 2010, 35:1322-1328. 10.1183/09031936.00092309

32. Dawson JK, Fewins HE, Desmond J, Lynch MP, Graham DR: Fibrosing alveolitis in patients with rheumatoid arthritis as assessed by high resolution computed tomography, chest radiography, and pulmonary function tests. Thorax. 2001, 56:622-627.

33. Raghu G, Remy-Jardin M, Myers JL, et al.: Diagnosis of idiopathic pulmonary fibrosis. An official ATS/ERS/JRS/ALAT clinical practice guideline. Am J Respir Crit Care Med. 2018, 198:44-68. 10.1164/rccm.201807-1255ST

34. Travis WD, Costabel U, Hansell DM, et al.: An official American Thoracic Society/European Respiratory Society statement: update of the international multidisciplinary classification of the idiopathic interstitial pneumonias. Am J Respir Crit Care Med. 2013, 188:733-748. 10.1164/rccm.201308-1483ST

35. Ciancio N, Pavone M, Torrisi SE, et al.: Contribution of pulmonary function tests (PFTs) to the diagnosis and follow up of connective tissue diseases. Multidiscip Respir Med. 2019, 14:17. 10.1186/s40248-019-0179-2

36. Garcia JG, Parhami N, Killam D, Garcia PL, Keogh BA: Bronchoalveolar lavage fluid evaluation in rheumatoid arthritis. Am Rev Respir Dis. 1986, 133:450-454. 10.1164/arrd.1986.133.3.450

37. Gochuico BR, Avila NA, Chow CK, et al.: Progressive preclinical interstitial lung disease in rheumatoid arthritis. Arch Intern Med. 2008, 168:159-166. 10.1001/archinternmed.2007.59

38. Kim EJ, Collard HR, King TE Jr: Rheumatoid arthritis-associated interstitial lung disease: the relevance of histopathologic and radiographic pattern. Chest. 2009, 136:1397-1405. 10.1378/chest.09-0444

39. Lee HK, Kim DS, Yoo B, Seo JB, Rho JY, Colby TV, Kitaichi M: Histopathologic pattern and clinical features of rheumatoid arthritis-associated interstitial lung disease. Chest. 2005, 127:2019-2027. 10.1378/chest.127.6.2019

40. Olson AL, Swigris JJ, Sprunger DB, et al.: Rheumatoid arthritis-interstitial lung disease-associated mortality . Am J Respir Crit Care Med. 2011, 183:372-378. 10.1164/rccm.201004-0622OC

41. Bongartz T, Nannini C, Medina-Velasquez YF, et al.: Incidence and mortality of interstitial lung disease in rheumatoid arthritis: a population-based study. Arthritis Rheum. 2010, 62:1583-1591. 10.1002/art.27405

42. Shaw M, Collins BF, Ho LA, Raghu G: Rheumatoid arthritis-associated lung disease . Eur Respir Rev. 2015, 24:1-16. 10.1183/09059180.00008014

43. Hassan WU, Keaney NP, Holland CD, Kelly CA: Bronchial reactivity and airflow obstruction in rheumatoid arthritis. Ann Rheum Dis. 1994, 53:511-514. 10.1136/ard.53.8.511

44. Wilczynska MM, Condliffe AM, McKeon DJ: Coexistence of bronchiectasis and rheumatoid arthritis: revisited. Respir Care. 2013, 58:694-701. 10.4187/respcare.01857

45. Geri G, Dadoun S, Bui T, Del Castillo Pinol N, Paternotte S, Dougados M, Gossec L: Risk of infections in 
bronchiectasis during disease-modifying treatment and biologics for rheumatic diseases. BMC Infect Dis. 2011, 11:304. 10.1186/1471-2334-11-304

46. Brazeau-Lamontagne L, Charlin B, Levesque RY, Lussier A: Cricoarytenoiditis: CT assessment in rheumatoid arthritis. Radiology. 1986, 158:463-466. 10.1148/radiology.158.2.3941873

47. Hayakawa H, Sato A, Imokawa S, Toyoshima M, Chida K, Iwata M: Bronchiolar disease in rheumatoid arthritis. Am J Respir Crit Care Med. 1996, 154:1531-1536. 10.1164/ajrccm.154.5.8912776

48. Lin E, Limper AH, Moua T: Obliterative bronchiolitis associated with rheumatoid arthritis: analysis of a single-center case series. BMC Pulm Med. 2018, 18:105. 10.1186/s12890-018-0673-X

49. Avnon LS, Abu-Shakra M, Flusser D, Heimer D, Sion-Vardy N: Pleural effusion associated with rheumatoid arthritis: what cell predominance to anticipate?. Rheumatol Int. 2007, 27:919-925. 10.1007/s00296-0070322-9

50. Corcoran JP, Ahmad M, Mukherjee R, Redmond KC: Pleuro-pulmonary complications of rheumatoid arthritis. Respir Care. 2014, 59:55-59. 10.4187/respcare.02597

51. Balbir-Gurman A, Yigla M, Nahir AM, Braun-Moscovici Y: Rheumatoid pleural effusion. Semin Arthritis Rheum. 2006, 35:368-378. 10.1016/j.semarthrit.2006.03.002

52. Jones FL Jr, Blodgett RC Jr: Empyema in rheumatoid pleuropulmonary disease. Ann Intern Med. 1971, 74:665-671. 10.7326/0003-4819-74-5-665

53. Farquhar H, Vassallo R, Edwards AL, Matteson EL: Pulmonary complications of rheumatoid arthritis. Semin Respir Crit Care Med. 2019, 40:194-207. 10.1055/s-0039-1683995

54. Zrour SH, Touzi M, Bejia I, et al.: Correlations between high-resolution computed tomography of the chest and clinical function in patients with rheumatoid arthritis: prospective study in 75 patients. Joint Bone Spine. 2005, 72:41-47. 10.1016/j.jbspin.2004.02.001

55. Makol A, Crowson CS, Wetter DA, Sokumbi O, Matteson EL, Warrington KJ: Vasculitis associated with rheumatoid arthritis: a case-control study. Rheumatology (Oxford). 2014, 53:890-899. 10.1093/rheumatology/ket475

56. Schaeffer MR, Molgat-Seon Y, Ryerson CJ, Guenette JA: Supplemental oxygen and dypsnoea in interstitial lung disease: absence of evidence is not evidence of absence. Eur Respir Rev. 2017, 26:170033. 10.1183/16000617.0033-2017

57. O'Dwyer DN, Armstrong ME, Cooke G, Dodd JD, Veale DJ, Donnelly SC: Rheumatoid arthritis (RA) associated interstitial lung disease (ILD). Eur J Intern Med. 2013, 24:597-603. 10.1016/j.ejim.2013.07.004

58. Iqbal K, Kelly C: Treatment of rheumatoid arthritis-associated interstitial lung disease: a perspective review. Ther Adv Musculoskelet Dis. 2015, 7:247-267. 10.1177/1759720X15612250

59. Hallowell RW, Horton MR: Interstitial lung disease in patients with rheumatoid arthritis: spontaneous and drug induced. Drugs. 2014, 74:443-450. 10.1007/s40265-014-0190-z

60. Song JW, Lee HK, Lee CK, Chae EJ, Jang SJ, Colby TV, Kim DS: Clinical course and outcome of rheumatoid arthritis-related usual interstitial pneumonia. Sarcoidosis Vasc Diffuse Lung Dis. 2013, 30:103-112.

61. Raghu G, Depaso WJ, Cain K, et al.: Azathioprine combined with prednisone in the treatment of idiopathic pulmonary fibrosis: a prospective double-blind, randomized, placebo-controlled clinical trial. Am Rev Respir Dis. 1991, 144:291-296. 10.1164/ajrccm/144.2.291

62. Martinez FJ, de Andrade JA, Anstrom KJ, King TE Jr, Raghu G: Randomized trial of acetylcysteine in idiopathic pulmonary fibrosis. N Engl J Med. 2014, 370:2093-2101. 10.1056/NEJMoa1401739

63. Allison AC: Mechanisms of action of mycophenolate mofetil . Lupus. 2005, 14:2-8. 10.1191/0961203305lu2109oa

64. Fischer A, Brown KK, Du Bois RM, et al.: Mycophenolate mofetil improves lung function in connective tissue disease-associated interstitial lung disease. J Rheumatol. 2013, 40:640-646. 10.3899/jrheum.121043

65. Tzouvelekis A, Galanopoulos N, Bouros E, et al.: Effect and safety of mycophenolate mofetil or sodium in systemic sclerosis-associated interstitial lung disease: a meta-analysis. Pulm Med. 2012, 2012:143637. $10.1155 / 2012 / 143637$

66. Conway R, Low C, Coughlan RJ, O'Donnell MJ, Carey JJ: Methotrexate and lung disease in rheumatoid arthritis: a meta-analysis of randomized controlled trials. Arthritis Rheumatol. 2014, 66:803-812. 10.1002/art.38322

67. Chikura B, Sathi N, Lane S, Dawson JK: Variation of immunological response in methotrexate-induced pneumonitis. Rheumatology (Oxford). 2008, 47:1647-1650. 10.1093/rheumatology/ken356

68. Hou J, Ma T, Cao H, et al.: TNF- $\alpha$-induced NF-KB activation promotes myofibroblast differentiation of LRMSCs and exacerbates bleomycin-induced pulmonary fibrosis. J Cell Physiol. 2018, 233:2409-2419. $10.1002 /$ jcp. 26112

69. Mori S, Imamura F, Kiyofuji C, Sugimoto M: Development of interstitial pneumonia in a rheumatoid arthritis patient treated with infliximab, an anti-tumor necrosis factor alpha-neutralizing antibody. Mod Rheumatol. 2006, 16:251-255. 10.1007/s10165-006-0491-5

70. Lindsay K, Melsom R, Jacob BK, Mestry N: Acute progression of interstitial lung disease: a complication of etanercept particularly in the presence of rheumatoid lung and methotrexate treatment. Rheumatology (Oxford). 2006, 45:1048-1049. 10.1093/rheumatology/kel090

71. Schoe A, van der Laan-Baalbergen NE, Huizinga TW, Breedveld FC, van Laar JM: Pulmonary fibrosis in a patient with rheumatoid arthritis treated with adalimumab. Arthritis Rheum. 2006, 55:157-159. 10.1002/art.21716

72. Hadjinicolaou AV, Nisar MK, Bhagat S, Parfrey H, Chilvers ER, Ostör AJ: Non-infectious pulmonary complications of newer biological agents for rheumatic diseases--a systematic literature review. Rheumatology (Oxford). 2011, 50:2297-2305. 10.1093/rheumatology/ker289

73. Perez-Alvarez R, Perez-de-Lis M, Diaz-Lagares C, et al.: Interstitial lung disease induced or exacerbated by TNF-targeted therapies: analysis of 122 cases. Semin Arthritis Rheum. 2011, 41:256-264. 10.1016/j.semarthrit.2010.11.002

74. Dixon WG, Hyrich KL, Watson KD, Lunt M, Symmons DP: Influence of anti-TNF therapy on mortality in patients with rheumatoid arthritis-associated interstitial lung disease: results from the British Society for Rheumatology Biologics Register. Ann Rheum Dis. 2010, 69:1086-1091. 10.1136/ard.2009.120626 


\section{Cureus}

75. Vassallo R, Matteson E, Thomas CF Jr: Clinical response of rheumatoid arthritis-associated pulmonary fibrosis to tumor necrosis factor-alpha inhibition. Chest. 2002, 122:1093-1096. 10.1378/chest.122.3.1093

76. Atkins SR, Turesson C, Myers JL, Tazelaar HD, Ryu JH, Matteson EL, Bongartz T: Morphologic and quantitative assessment of CD20+ B cell infiltrates in rheumatoid arthritis-associated nonspecific interstitial pneumonia and usual interstitial pneumonia. Arthritis Rheum. 2006, 54:635-641. $10.1002 /$ art.21758

77. Becerra E, Cambridge G, Leandro M: FRI0228 safety and efficacy of rituximab in patients with rheumatoid arthritis and lung involvement. Ann Rheum Dis. 2013, 72:A450. 10.1136/annrheumdis-2013-eular.1355

78. Richardson CB, Singer JP: Lung transplantation for scleroderma-related lung disease . Curr Respir Care Rep. 2014, 3:79-87. 10.1007/s13665-014-0080-6

79. Wu C, Lin H, Zhang X: Inhibitory effects of pirfenidone on fibroblast to myofibroblast transition in rheumatoid arthritis-associated interstitial lung disease via the downregulation of activating transcription factor 3 (ATF3). Int Immunopharmacol. 2019, 74:105700. 10.1016/j.intimp.2019.105700

80. Doyle TJ, Lee JS, Dellaripa PF, et al.: A roadmap to promote clinical and translational research in rheumatoid arthritis-associated interstitial lung disease. Chest. 2014, 145:454-463. 10.1378/chest.13-2408 